\title{
Prevalence and Antimicrobial Susceptibility Pattern of Methicillin-resistant Staphylococcus aureus [MRSA] isolates at a Tertiary Care Hospital in Agra, North India - A systemic annual review
}

\author{
Ankur Goyal $^{1}$, Manish Kumar Diwakar ${ }^{2}$, Suneel Bhooshan ${ }^{3}$, Sapna Goyal ${ }^{4}$, \\ Arti Agrawal ${ }^{5}$ \\ 1, 3-5 Dept. of Microbiology, S.N. Medical College, Agra, India \\ ${ }^{2}$ Dept. of Microbiology, M.L.B. Medical College, Jhansi, India
}

\begin{abstract}
A total 1163(10.12\%) staphylococcus aureus were isolated from 11496 clinical specimens (2009 2012) among which 379 (32.6\%) were MRSA. In our retrospective study, we found a continuous increase in MRSA prevalence over the time which was $16.5 \%$ in 2009; increased to $22.2 \%$ in $2010,34.6 \%$ in 2011 and $37.5 \%$ in 2012? The average MRSA prevalence observed during this period was $27.7 \%$. The overall MRSA prevalence was $3.3 \%$ among all clinical specimens tested. No resistance was seen for Vancomycin, Teicoplanin and Linozolid. The highest sensitivity was observed by Doxycyclin (79.04\%) \& Amikacin (96\%) where as the least was by erythromycin (21.18\%) \& Chloramphenicol (52.2\%) for MRSA and MSSA isolates respectively. Females were more prevalent (62\%) as compared to males (38\%) for MRSA infections.
\end{abstract}

Keywords: Antimicrobial Sensitivity, disc diffusion, Methicillin resistant Staphylococcus aureus [MRSA], Methicillin sensitive Staphylococcus aureus [MSSA],

\section{Introduction}

Staphylococcus aureus is the most common human bacterial pathogen and is an important cause of skin and soft tissue infections, endovascular infections, pneumonia, tonsillitis, pharyngitis, septic arthritis, endocarditis, enterocolitis, osteomyelitis, meningitis, Toxic shock syndrome, sepsis, etc. Due to inappropriate use of antibiotics, the resistance in these strains is increasing worldwide. Methicillin-resistant Staphylococcus aureus (MRSA) are resistant to all available penicillins and other Beta -lactam antimicrobial drugs. Methicillinresistant S. aureus was first observed in 1961 after methicillin was introduced into clinical use in 1960 and since that time MRSA emerged as an important nosocomial pathogen worldwide.

In India, Methicillin-resistant Staphylococcus aureus (MRSA) represents a challenge for all healthcare institutions. Previously it was limited to large institutions; now quite common in all hospital settings $[1,2]$. MRSA is now one of the commonest nosocomial pathogens, and S. aureus asymptomatically colonizing healthcare workers are the major sources of MRSA in the hospital environment [3]. The prevalence of MRSA strains has increased worldwide. This increase has been associated with the reorganization of new community-associated MRSA (CA-MRSA) strains, which has increased the disease burden in general population with or without exposure to the health care environment [4]. Beside this, prolonged hospitalization, use of invasive medical devices, healthcare workers, suppressed immune system, prolonged use of antimicrobials, living in crowded or unsanitary conditions are some risk factors for MRSA infection.

According to European Antimicrobial Resistance Surveillance System report, there has been shown significant increases in methicillin resistance Staphylococcus aureus between 1999 and 2002 with the highest incidence of $44 \%$ in Greece and lowest of $0.5 \%$ in Iceland [5]. Whereas in the similar study by Brog M et al. [6] in 2006 in Jordan, $65 \%$ MRSA prevalence was reported. According to National Nosocomial Infection Surveillance System (NNIS) report, 50\% of hospital acquired infections in ICUs in the USA are due to MRSA [7].

The growing problem in the Indian scenario is that MRSA prevalence is rapidly increasing with the time. In a study conducted in Indore the MRSA has been increased to $80.83 \%$ in 1999 which was $12 \%$ in 1992 [8]. The same was also observed in Uttar Pradesh where the incidence of MRSA was as low as $6.9 \%$ in 1988 which raised to $32.8 \%$ in 1994 [9] and 59.3\% in 2008 [10]. In addition to the terrible situation, MRSA strains are important for their resistance to many other commonly used antibiotics and the emergence of resistance to Vancomycin, the drug which is considered as last resort for these strains. It is essential to control the emerging problem of Vancomycin resistance and multidrug resistance in MRSA strains. Reports of emergence of Vancomycin resistance in S. aureus from India (Assadullah et al 2003; Tiwari and Sen 2006; Saha et al 2008) further justify this necessity. 
We must have to know the MRSA prevalence and the current antimicrobial profile in our population so that appropriate measures can be formulate for these infections and irrational use of vancomycin and other antimicrobials could be minimize. Therefore, we planned this study to determine the prevalence of MRSA isolates and their antimicrobial profile from our hospital so as to formulate an appropriate as well as cost effective empirical therapy and make better hospital infection control policies.

\section{Materials And Methods}

This retrospective analysis included 11496 different clinical samples such as pus and wound swab, urine, blood, CSF, body fluids, sputum, throat swab, ear swab, vaginal swab, catheter tip, corneal fluid, , etc obtained from OPD \& IPD of different departments from 2009 to 2012. All specimens, except urine for which cystine lactose electrolyte deficient agar (CLED Agar) was used, were cultured on blood agar and MacConkey agar (HiMedia, New Delhi, India).

S. aureus were identified and differentiated from related organisms as per conventional methods on the basis of colony morphology, Gram staining, catalase test, slide and tube coagulase, mannitol fermentation and DNase production following the standard procedures.[11]

The antibiotic susceptibility patterns of all the Staphylococcus aureus isolates were determined by Kirby Bauer disc diffusion method against the following antibiotics:

Ampicillin (10mcg), Amikacin (30mcg), Clindamycin (2mcg), Cefoxitin (30mcg), Chloramphenicol (30 mcg), Co-Trimoxazole $(1.25 / 23.75 \mathrm{mcg})$, Ciprofloxacin $(5 \mathrm{mcg})$, Doxycyclin (30 mcg), Erythromycin $(15 \mathrm{mcg})$, Gentamycin $(10 \mathrm{mcg})$, Linozolid (30 mcg), Teicoplanin $(30 \mathrm{mcg})$ and Vancomycin $(30 \mathrm{mcg})$

The test was performed on Mueller-Hinton agar, and interpreted after 24 hours of incubation at $37^{\circ} \mathrm{C}$ Methicillin resistance was detected by Cefoxitin disc diffusion test. The inhibition zone diameters were measured around each disc and were interpreted according to the Clinical Laboratory Standards Institute guidelines $[12,13,14,15]$.

S. aureus ATCC 25923 (MSSA strain) and ATCC 43300 (MRSA strain) were used for the quality control of all the tests. Data analysis was done by the help of Microsoft excel 2007 software.

\section{Results}

In the present retrospective study, 11496 different clinical specimens were collected such as pus and wound swab, urine blood, CSF, body fluids, sputum, throat swab, ear swab, vaginal swab, catheter tip, corneal fluid, etc. from 2009 to 2012. A total 1163 isolates were identified as staphylococcus aureus in which 379 (32.6\%) were Methicillin Resistant Staphylococcus aureus. In this study, we observed that there is a continuous increase in prevalence of MRSA over years. In 2009 it was $16.5 \%$, raised to $37.5 \%$ in 2012 [Fig-1]. The prevalence was higher in females $(235 ; 62 \%)$ than males $(144 ; 38 \%)$. Out of all MRSA isolates, the highest prevalence was observed in pus samples $(250 ; 66.03 \%)$ followed by urine $(43 ; 11.45 \%)$, Blood \& tips (35; $9.16 \%)$, sputum $(30 ; 8.02 \%)$, swab $(9 ; 2.29 \%)$, CSF $(9 ; 2.29 \%) \&$ other body fluids $(3 ; 0.76 \%)$. The least prevalence was in corneal fluid $(0.4 \%)$.

Most of the MRSA strains were isolated in age group of 20-40 years which accounts for $51 \%$ of the total MRSA prevalence followed by 0-20 age group patients (26\%), 40-60 age group (18\%) and the least was found in 60-80 years age group (5\%). The prevalence of MRSA in OPD was 43.89\% (166) and 56\% (212) was in IPD. The highest prevalence was observed in the patients of gynecology department (42.72\%) followed by Intensive care unit $(12.44 \%)$, Neonatal ICU (12.24\%), Medicine (10.88\%), Chest \& Tuberculosis $(9.48 \%)$, Pediatrics $(8.84 \%)$, ENT department $(2.04 \%)$ and Ophthalmology department (1.36\%). All the MRSA isolates (100\%) were Resistant to Ampicillin and all were sensitive to Vancomycin, Teicoplanin and Linozolid. Doxycyclin (79.04\%), Amikacin (77.71\%) and Clindamycin (52.4\%) showed good efficacy where as Gentamycin , Chloramphenicol, Ciprofloxacin, Co-trimoxazole and Erythromycin showed less sensitivity as $48.77 \%, 42.36 \%, 41.95 \%, 21.18 \%$ and $20.25 \%$ respectively against MRSA strains in our in-vitro study. The sensitivity pattern of MSSA was quite differ from the MRSA strains. Amikacin showed the highest sensitivity (96\%) against MSSA strains followed by Gentamycin (86.05\%), Doxycyclin (84.8\%), Ciprofloxacin (79.5\%), Clindamycin (77.01\%), Erythromycin (67.35\%), Co-trimoxazole (56.36\%) and Chloramphenicol $(52.2 \%)$. The least sensitivity was showed by erythromycin $(21.18 \%)$ and Chloramphenicol $(52.2 \%)$ against MRSA and MSSA strains respectively. Since January 2009 to June 2012, we observed a continuous decrease in sensitivity of Co-trimoxazole, Ciprofloxacin, Amikacin and Doxycyclin to MRSA strains in our population [Fig-2]. 


\section{Tables And Figures}

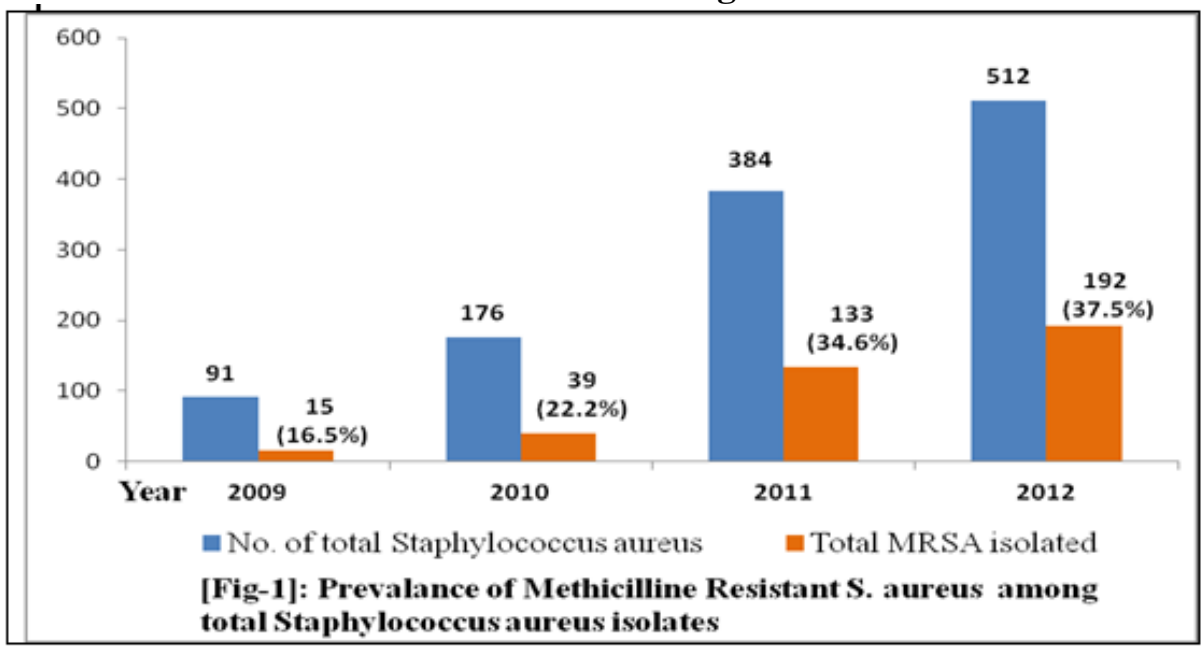

\begin{tabular}{|c|c|c|c|c|}
\hline \multirow{2}{*}{ Antibiotics } & 2009 & 2010 & 2011 & 2012 \\
\hline & $\%$ Sensitivity & $\%$ Sensitivity & $\%$ Sensitivity & $\%$ Sensitivity \\
\hline Ampicillin & 0.00 & 0.00 & 0.00 & 0.00 \\
\hline Amikacin & 80.00 & 79.49 & 76.69 & 74.67 \\
\hline Clindamycin & 53.33 & 51.28 & 55.64 & 49.33 \\
\hline Chloramphenicol & 46.67 & 43.59 & 45.86 & 33.33 \\
\hline Co-trimoxazole & 26.67 & 23.08 & 20.30 & 14.67 \\
\hline Doxycyclin & 80.00 & 84.62 & 78.20 & 73.33 \\
\hline Erythromycin & 26.67 & 20.51 & 21.80 & 12.00 \\
\hline Gentamycin & 53.33 & 58.97 & 48.12 & 34.67 \\
\hline Ciprofloxacin & 46.67 & 43.59 & 42.86 & 34.67 \\
\hline Teicoplanin & 100 & 100 & 100 & 100 \\
\hline Linozolid & 100 & 100 & 100 & 100 \\
\hline Vancomycin & 100 & 100 & 100 & 100 \\
\hline
\end{tabular}

\section{Discussion}

Staphylococcus aureus is a major virulent human pathogen that is commonly isolated from hospitals and community. Increased antimicrobial resistance for such an organism is, therefore a cause of concern. As use of new antistaphylococcal agents have become common, there has been a rise in resistance to these newer antibiotics. In recent years the prevalence of MRSA is continuously increasing in hospital as well as in community.

In the present retrospective study during 2009 to 2012; we found high prevalence of MRSA isolates in our clinical specimen. There is more than two folds increase in MRSA prevalence from 2009 (16.5\%) to 2012 (37.5\%). Our data suggest a continuous increase in prevalence of MRSA in our set-up [Fig-1]. In a similar study conducted in J.N. Medical College, Wardha (Maharashtra), an increased incidence of MRSA as 51.8\% in 2010 was reported by Basak et al [16] which was 30.6\% in 1997. Tiemersma et al. [5] also reported a significant increase in methicillin resistance in clinical isolates of S. aureus between 1999 and 2002 in European countries. At the beginning of their study the prevalence of $9.4 \%, 30.5 \%$ and $37 \%$ was increased to $19.2 \%, 44.5 \%$ and $48.6 \%$ in Germany, United Kingdom and in Greece respectively.

Our retrospective study showed $32.6 \%$ prevalence of MRSA in different clinical samples. Many reports showed the similar prevalence of MRSA from different places in India and surrounding ranging from $26.14 \%$ to $43 \%[3,10,17,18,19,20,21,22]$. However, the similar studies conducted in Uttar Pradesh, India showed the very high MRSA prevalence ranging from $54.85 \%$ by Anupurba $\mathrm{S}$ et al. in 2003 [23] to 59.3\% by Tiwari et al in 2006 [24] which is much higher than other places and the present study. This variation might be because of variation in antibiotic usage and infection control practices in different places or variation in patient and clinical specimens.

In our study there was no resistance observed for Vancomycin, Linezolid and Teicoplanin in MRSA as well as MSSA strains. Loveena Oberoi et al. [25] and Bharti Arora et al. [26] also found 100\% sensitivity for 
these drugs in their study in Punjab and Haryana. However, very few studies reported vancomycin resistance (VRSA) in MRSA strains [24,27].

Doxycyclin (79.04\%), Amikacin (77.71\%) and Clindamycin (52.4\%) showed good efficacy where as Gentamycin , Chloramphenicol, Ciprofloxacin, Co-trimoxazole and Erythromycin showed less sensitivity as $48.77 \%, 42.36 \%, 41.95 \%, 21.18 \%$ and $20.25 \%$ respectively against MRSA strains in our in-vitro study. In our study the least sensitivity was showed by erythromycin $(21.18 \%)$ and Chloramphenicol (52.2\%) against MRSA and MSSA strains respectively. However many studies from various places showed higher resistance percentage for these antibiotics in their MRSA isolates, in compared to our study [23, 24]. The reason for lesser resistance in our MRSA isolates may be due to the more number of community acquired isolates in our study. Other reason may be the difference in antibiotic prescription in various communities.

In a comparative sex wise study of MRSA prevalence, females $(62 \%)$ were more affected than male patient (38\%). The similar prevalence ratio (61:39) of female and male was also reported in Himachal Pradesh by Sharma \& Mall [28]. However just opposite to our finding Female and Male prevalence ratio was reported (40:60) by Rao BN et al. [29] in 2012 in their study conducted in Andhra Pradesh. The most affected age group was 20-40 years in our study. However Sharma \& mall [28] reported above 55 years and Dar JA et al [19] reported 45-65 years as the highest effected age groupIn the present study, the IPD cases (56\%) were more than OPD $(44 \%)$ cases. Among IPD, the highest prevalence was observed in gynecology department $(42.72 \%)$. This prevalence was significantly higher than other clinical departments. Moreover this prevalence was very much lower in ophthalmology (1.36\%) and ENT (2.04\%) patients. However various studies shows entirely different patient distribution among IPD \& OPD patients. Mallick SK and Basak S [16] reported 84.8\% MRSA from IPD patient in which $25.5 \%$ MRSA strains were isolated from the surgical ward and $23.4 \%$ from the Orthopedic ward, $11.7 \%$ from Medicine Ward and 9.7\% from Gynecology ward whereas Loveena et al. [25] reported maximum isolation of MRSA from Orthopedics ward (28.86\%), followed by Surgery (21.65\%), Medicine $(16.49 \%)$ and Gynecology (5.51\%). Similarly, in a study by Sanjana RK et al. [30], the majority of the samples were obtained from Surgery $(24 \%)$ and Orthopaedics units $(16 \%)$.

Among all clinical samples the highest prevalence (66.03\%) was seen in pus samples in this study. The same was also reported by Tiwari et al. [10] in Varanasi (42\%), Basak et al. [16] in Maharashtra (61.4\%), Dar JA et al. [19] in Aligarh (35.5\%) and Rao BN et al. [29] in Andhra Pradesh (64\%). As in the present study, a continuous increase in prevalence of MRSA is of great concern for our healthcare system, good hospital infection control policies may be a good barrier for these infections. Because the potential reservoirs of MRSA include infectious patients, hospital personnel and hospital environment, regular monitoring of antimicrobial sensitivity pattern of all clinical isolates, and continuous infection control measures like hand washing and other aseptic techniques will definitely control and decrease the incidence of MRSA as well as other multidrug resistant pathogen.

\section{Conclusion}

The antibiotic sensitivity pattern of MRSA for commonly used antibiotics is recognized to be diverse from region to region. Glycopeptides (Vancomycin and Teicoplanin) and Linozolid seem to be the only antimicrobial agent which showed $100 \%$ sensitivity and may be used as the drug of choice for treating MRSA infections. The most effective way to prevent MRSA infection is by performing regular surveillance of antibiotic profile of local staphylococcus isolates to formulate antibiotic policies and effective infection control practices. The usual hygienic methods such as hand disinfection after each contact with patients and the use of masks by all healthcare workers in hospitals should be implemented to reduce the chance of hospital acquired MRSA infections.

References

[1] Anupurba S, Sen MR, Nath G, Sharma BM, Gulati AK, Mohapatra TM. Prevalence of methicillin resistant Staphylococcus aureus in a tertiary referral hospital in eastern Uttar Pradesh. Indian J Med Microbiology. 2003. 21. 49-51.

[2] Salaria M, Singh M. Methicillin resistant Staphylococcus aureus. Indian Pediatrics. 2001. 38. 29-36.

[3] Pai V, Rao IR, Rao SP. Prevalence and Antimicrobial Susceptibility Pattern of Methicillin-resistant Staphylococcus Aureus [MRSA] Isolates at a Tertiary Care Hospital in Mangalore, South India. Journal of Laboratory Physicians. 2010. 2(2). 82-84.

[4] David MZ and Daum RS. Community-Associated Methicillin-Resistant Staphylococcus aureus: Epidemiology and Clinical Consequences of an Emerging Epidemic. Clinical microbiology review. 2010; 23(3): 616-687.

[5] Tiemersma EW, Bronzwear LAM, Lyytikainen O, degener JE, Schrijnemakers P, Bruinsma N, Monan J, Witte W, Grundmaun H. Methicillin resistant Staphylococcus aureus in Europe 1999-2002. • www.cdc.gov/eid • Emerge infect Disease. 2004; 10(9):16271634.

[6] Borg M, Scicluna E, De Kraker M, Van de Sande-Bru insma N, Tiemersma E, Gur D et al. Antibiotic resistance in the southeastern Mediterranean - preliminary results from the AR Medical project (Staphylococcus aureus, European Surveillance). $2006 ; 20(11): 7$

[7] Salgado CD, Farr BM, Calfee DP. Community acquired methicillin resistant Staphylococcus aureus: A meta-analysis of prevalence and risk factors. Clin. Infectious Disease. 2003; 131-139.

[8] Verma S, Joshi S, Chitnis V, Hemwani N, Chitnis D. Growing problem of methicillin resistant staphylococci - Indian scenario. Indian J Med Sciences. 2000; 54:535-40. 
[9] Mathur SK, Singal, S, Prasad, KN, Kishore J, Ayyagiri A. Prevalence of MRSA in tertiary care hospital. Indian J Medical Microbiology. 1994;12: 96-101.

[10] Tiwari HR, Sapkota D, Sen MR. High prevalence of multidrug-resistant MRSA in a tertiary care hospital of northern India. Infection and Drug Resistance. 2008:1 57-61.

[11] Washington CW Jr, Stephen DA, William MJ, et al. Gram positive cocci. Koneman's Color Atlas and Textbook of Diagnostic Microbiology. 6th edition. Philadelphia: Lippincott Williams \& Williams. 2006; 12: 623-71.

[12] Clinical and Laboratory Standards Institute [CLSI]. Performance Standards for Antimicrobial disk Susceptibility TestingApproved Standard.10th edition. M02-A10. 2009; 29(1)

[13] Clinical and Laboratory Standards Institute [CLSI]. Performance Standards for Antimicrobial disk Susceptibility TestingApproved Standard.10th edition. M100-S20. 2010; 30(1)

[14] Clinical and Laboratory Standards Institute [CLSI]. Performance Standards for Antimicrobial Susceptibility Testing. Twenty-First Informational Supplement. M100-S21. 2011; 31(1)

[15] Clinical and Laboratory Standards Institute [CLSI]. Performance Standards for Antimicrobial Susceptibility Testing- Approved Standard. 11th edition. M02-A11. 2012; 32(1)

[16] Mallick SK and Basak S. MRSA- too many hurdles to overcome: a study from Central India. Tropical Doctor. 2010; 40: 108-110.

[17] Rajaduraipandi K, Mani KR, Panneerselvam K, Mani M, Manikandan P. Prevalence and antimicrobial susceptibility pattern of methicillin resistant Staphylococcus aureus: A multicenter study. Indian Journal of Medical microbiology. 2006; 24:34-38.

[18] Tsering DC, Pal R, Kar S. Methicillin-resistant Staphylococcus Aureus: Prevalence and current susceptibility pattern in Sikkim. J Global Infectious Disease. 2011; 3:9-13.

[19] Dar JA, Thoker MA, Khan JA, Ali A, Khan MA, Rizwan M, et al. Molecular epidemiology of clinical and carrier strains of methicillin resistant Staphylococcus Aureus (MRSA) in the hospital settings of north India. Ann Clin Microbiol Antimicrob. 2006; 5:22.

[20] Dalela G, Gupta S, Jain DK, Mehta P. Antibiotic Resistance Pattern in Uropathogens at a Tertiary Care Hospital at Jhalawar with Special Reference to ESbl, AmpC $\beta$-lactamase and MRSA Production. Journal of Clinical and Diagnostic Research. 2012 ; 6(4): 645-651.

[21] Perwaiz S, Barakzi Q, Farooqi BJ, Khursheed N, Sabir N. Antimicrobial susceptibility pattern of clinical isolates of Methicillin Resistant Staphylococcus aureus. J Pak Med Association. 2007; 57(1): 2-4.

[22] Kumari N, Mohapatra TM, Singh YI. Prevalence of Methicillin-resistant Staphylococcus aureus (MRSA) in a Tertiary-Care Hospital in Eastern Nepal. J Nepal Med Association. 2008; 47(170): 53-6.

[23] Anupurba S, Sen MR, Nath G, Sharma BM, Gulati AK, Mohapatra TM. Prevalence of methicillin resistant Staphylococcus aureus in tertiary referral hospital in Eastern Uttar Pradesh. Indian J Med Microbiology. 2003; 21(1):49-51.

[24] Tiwari HK, Sen MR. Emergence of vancomycin resistant Staphylococcus aureus (VRSA) from a tertiary care hospital from northern part of India. BMC Infect Dis. 2006; 6:156.

[25] Oberoi L, Kaur R, Aggarwal A. Prevalence and antimicrobial susceptibility pattern of Methicillin resistant Staphylococcus aureus (MRSA) in a rural tertiary care hospital in north India. International Journal of Applied Biology and Pharmaceutical Technology. 2012; 3(1): 200-205.

[26] Arora B, Ranjan KP, Arora DR. Prevalence of Methicillin Resistant Staphylococcus aureus in Post Operative Infections in a Referral Hospital in Haryana, India. J Infectious Disease Antimicrobial Agents. 2008; 25:123-7.

[27] Menezes GA, Harish BN, Sujatha S, Vinothini K, Parija SC. Emergence of vancomycin-intermediate Staphylococcus species in southern India. J of Medical Microbiology. 2008; 57:911-912.

[28] Sharma S and Mall A. The prevalence, Antibiogram and characterization Methicillin resistant Staphylococcus aureus among the patients from the Doon Valley hospitals. African Journal of Microbiology Research. 2011; 5(21): 3446-3451.

[29] Rao BN, Srinivas B. A prospective study of Methicillin resistant staphylococcus aureus (MRSA) in a teaching hospital of rural setup. Journal of Pharmaceutical and Scientific Innovation. 2012; 1(2): 37-40.

[30] Sanjana RK, Shah R, Chaudhary N, Singh YI. Prevalence and antimicrobial susceptibility pattern of Methicillin resistant Staphylococcus aureus (MRSA) in CMS-teaching hospital: a preliminary report. Journal of College of Medical Sciences. 2010; 6(1):1-6. 